\title{
A New Two-stage Photovoltaic GMPPT Method under Partially Shaded Conditions
}

\author{
Shuran Jia ${ }^{1, a^{*}}$, Qingyong Zhang ${ }^{1, b}$ and Yang Fang ${ }^{1, c}$ \\ ${ }^{1}$ School of Automation, Wuhan University of Technology, 122 Luoshi Road,Wuhan,Hubei, \\ P.R.China \\ ªwinme2000@whut.edu.cn, bqyzhang@whut.edu.cn, ’superminions@whut.edu.cn
}

\begin{abstract}
Keywords: Photovoltaic; Solar Energy; Partially Shaded Conditions; GMPPT; MPPT; PV System. Abstract. In a photovoltaic (PV) system that consists of multiple series-connected PV modules with bypass diode, multiple peak points could exist in the P-V curve of the PV system under partially shaded conditions (PSC), causing conventional MPPT methods sometimes unable to track the true global maximum power point (GMPP). In view of this problem, we propose in this paper a new two-stage global maximum power point tracking (GMPPT) method based on the distribution characteristic of the GMPP voltage and the dividing point voltage. The method proposed finds all the possible peak power points and compares the power of those points to find the GMPP in the first stage. In the second stage, the method sets the operation point to the point of the maximum power found in the first stage and uses the hill climbing (HC) method to keep the operation point at that point. The method proposed is validated by simulation. Simulation result shows that this method can track the GMPP effectively.
\end{abstract}

\section{Introduction}

Solar energy is of great interest due to its abundance of energy source, almost zero pollution and the advancement in semiconductor and power electronic devices. Photovoltaic (PV) module is the most frequently used device to directly convert solar energy into electricity via photovoltaic effect. In a photovoltaic (PV) system that consists of multiple series-connected PV modules with bypass diodes, due to cloud or other factors, some PV modules in the system could be shaded, resulting in their lower irradiances received than those of the unshaded PV modules, which is called partially shaded condition (PSC). Under PSC, multiple peak points could exist in the power-voltage (P-V) curve of the PV system [1]. Among those peak points, the highest peak point is GMPP and the rest of the points are local maximum power points (LMPPs).

To maximize the power output of the PV system, the terminal voltage of the PV system should be kept at the GMPP voltage, which is done by MPPT controllers using MPPT methods. Hence the MPPT controllers are essential to PV power generation systems. There are many conventional MPPT methods such as hill climbing (HC) / perturb \& observe (P\&O) method and incremental conductance (IncCond) method [2]. Those methods including P\&O and InCond can track the MPP effectively only when single peak point exists in the P-V curve of the PV system. However, when multiple peak points occur, those methods may fail to track the GMPP but fall into the LMPP. Due to this, some methods for multiple-peak MPPT have been proposed. A two-stage MPPT proposed in [3] sweeps the P-V curve of the PV system to search for the GMPP in the first stage and use P\&O method to keep the operation point at the GMPP in the second stage. The method will back to the first stage if it notices a change in the power to ensure it can track the GMPP under varying conditions. However, this method needs to search many points to find the GMPP, which is time-consuming. A method that uses Fibonacci search algorithm to find the GMPP is proposed in [4], which reduces the number of the searching points. However, this algorithm is complicated and could be difficult to implement. Another two-stage GMPP method is proposed in [5], this method requires additional circuit to measure the open circuit voltage and short circuit current, which adds complexity to the hardware and the control algorithm. Reference [6] proposes a GMPP method based on artificial neural network. This method is fast in speed and can track the GMPP under various conditions. However, this method requires additional 
sensors installed on every PV module to measure the irradiance of each PV module, which adds cost to the system.

In this paper, we propose a new two-stage GMPPT method based on the distribution characteristic of the GMPP voltage, the dividing point voltage and the hill climbing (HC) method. Compared to the other methods mentioned above, this method is easy to implement and has good effectiveness in tracking the GMPP.

\section{The PV System}

In this paper, we use the model proposed in [7] to characterize the PV module. We construct a PV system that consists of 3 series-connected identical PV modules with bypass diodes as is shown in Fig. 1. The parameters of the PV module under $25^{\circ} \mathrm{C}$ and $1000 \mathrm{~W} / \mathrm{m}^{2}$ are shown in Table 1.

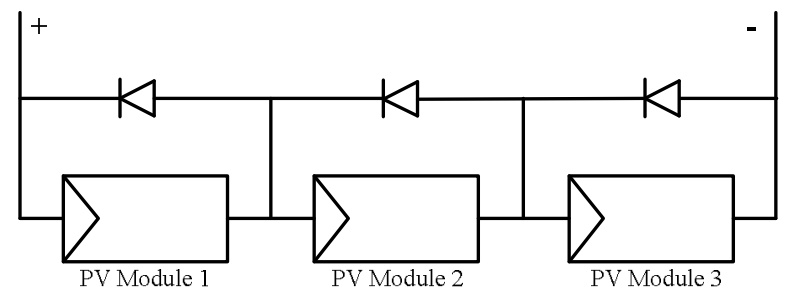

Fig. 1 PV System Configuration

Table 1 The Parameters of the PV Module

\begin{tabular}{cc}
\hline Parameter & Value \\
\hline Open circuit voltage (Voc) & $22 \mathrm{~V}$ \\
Short circuit current (Isc) & $8.58 \mathrm{~A}$ \\
Maximum power (Pmax) & $140 \mathrm{~W}$ \\
Maximum power point voltage (Vm) & $17.7 \mathrm{~V}$ \\
\hline
\end{tabular}

The Presence of Multiple Peak Points on the P-V Curve under PSC. To illustrate the existence of multiple peak points on the P-V curve of the PV system under PSC, we set 3 different irradiance patterns (I.P.) to imitate various shading patterns. In each pattern, there are various irradiances for PV module 1, PV module 2 and PV module 3 denoted by irr1, irr2 and irr3 respectively. In pattern 1, the irradiances are $[\operatorname{irr} 1, \operatorname{irr} 2, \operatorname{irr} 3]=\left[1000 \mathrm{~W} / \mathrm{m}^{2}, 1000 \mathrm{~W} / \mathrm{m}^{2}, 1000 \mathrm{~W} / \mathrm{m}^{2}\right]$, which are under uniform irradiance condition. In pattern 2, the irradiances are $[\operatorname{irr} 1, \operatorname{irr} 2, \operatorname{irr} 3]=\left[1000 \mathrm{~W} / \mathrm{m}^{2}, 500 \mathrm{~W} / \mathrm{m}^{2}\right.$, $\left.300 \mathrm{~W} / \mathrm{m}^{2}\right]$. In pattern 3 , the irradiances are $[\operatorname{irr} 1, \operatorname{irr} 2, \operatorname{irr} 3]=\left[1000 \mathrm{~W} / \mathrm{m}^{2}, 200 \mathrm{~W} / \mathrm{m}^{2}, 100 \mathrm{~W} / \mathrm{m}^{2}\right]$. The 3 patterns are input to the system respectively and the system is simulated to obtain the P-V curves under 3 various irradiance patterns. The results are shown in Fig. 2. From the results, it can be observed that there is only 1 peak point on the $\mathrm{P}-\mathrm{V}$ curve under irradiance pattern 1 and there are 3 peak points on the $\mathrm{P}-\mathrm{V}$ curves of the PV system under irradiance pattern 2 and 3. The GMPP is the second peak point under irradiance pattern 2 and the GMPP is the first peak point under irradiance pattern 3. Thus multiple peak points could exist on the P-V curve of the PV system under PSC. The simulation results also show that the GMPP could be the various peak points.

The Distribution of GMPP under Various Conditions. By changing the irradiance on each module in $50 \mathrm{~W} / \mathrm{m}^{2}$ steps from $50 \mathrm{~W} / \mathrm{m}^{2}$ to $1000 \mathrm{~W} / \mathrm{m}^{2}$, the GMPP under various irradiance patterns can be generated. Fig. 3 shows the distribution of GMPP obtained by simulation. From Fig. 3, it can be found that the GMPP voltages are only located in particular regions under various conditions, which are $[12 \mathrm{~V}, 17 \mathrm{~V}],[28 \mathrm{~V}, 36 \mathrm{~V}]$ and $[43 \mathrm{~V}, 56 \mathrm{~V}]$. The centers of the regions are approximately Eq. 1, where $\mathrm{k}$ is a constant between $0.7 \sim 0.8$, Voc is the open circuit voltage of PV module and $\mathrm{n}$ is a positive integer from 1 to the number of series connected PV modules.

$$
\mathrm{V}=\mathrm{k} \cdot \mathrm{n} \cdot \mathrm{Voc}
$$




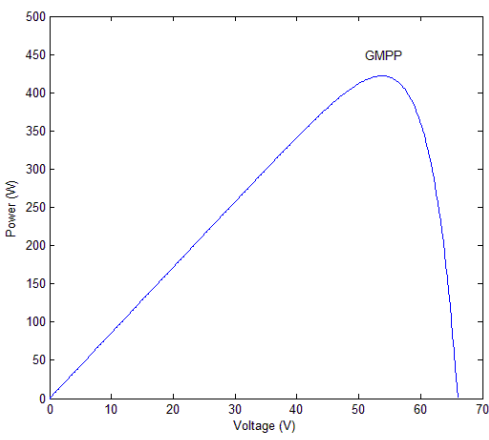

(a) under I.P. 1

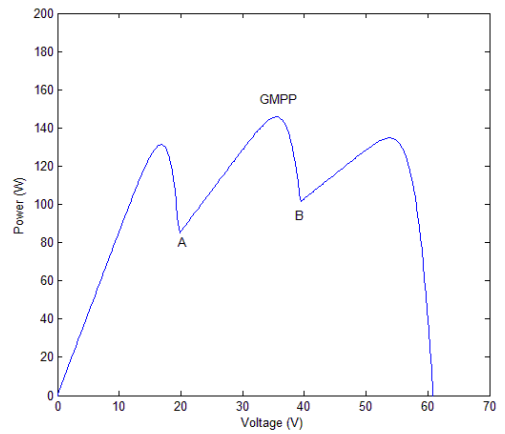

(b) under I.P. 2

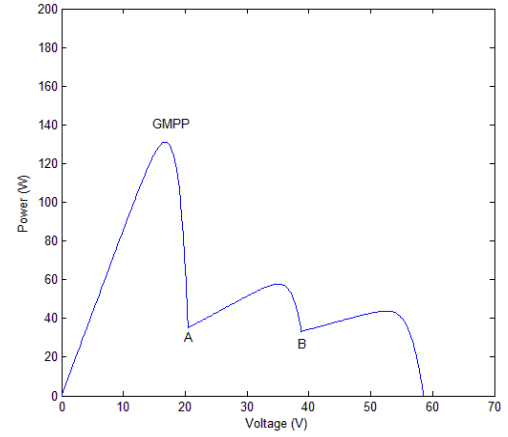

(c) under I.P. 3

Fig. 2 the P-V Curve of the PV System under Various Irradiance Patterns

The Distribution of Dividing Points on the P-V Curve under Various Conditions. The dividing points are the points that divide the $\mathrm{P}-\mathrm{V}$ curve into several hill-shaped curve parts. For instance, the points A and B in the Fig. 2(b) and Fig. 2(c) are the dividing points of the P-V curve. It's obvious that the neighbor points of a dividing point are higher than the dividing point and there is only one maxima point in each of the parts that the dividing points divide the $\mathrm{P}-\mathrm{V}$ curve into. Among the maxima points of each part, there is one maxima point that is GMPP and the others are LMPPs. For the proceeding studies, the dividing points under various conditions are obtained by simulation. The results are shown in Fig. 4. It can be seen that the voltages of the dividing points are also located in particular regions under various conditions, which are $[15 \mathrm{~V}, 21 \mathrm{~V}]$ and $[32 \mathrm{~V}, 44 \mathrm{~V}]$. And the dividing-point-free regions (regions that never contain any dividing point under any irradiance conditions) are $[0,15 \mathrm{~V}],[21 \mathrm{~V}$, $32 \mathrm{~V}]$ and $[44 \mathrm{~V}, 66 \mathrm{~V}]$. For the P-V curve with $\mathrm{N}$ peak points, there are $\mathrm{N}-1$ dividing points on the $\mathrm{P}-\mathrm{V}$ curve which divide the $\mathrm{P}-\mathrm{V}$ curve into $\mathrm{N}$ dividing-point-free regions.

Based on the distribution of dividing points on the P-V curve under various conditions, we can propose a new GMPPT method that can be applied to various conditions.

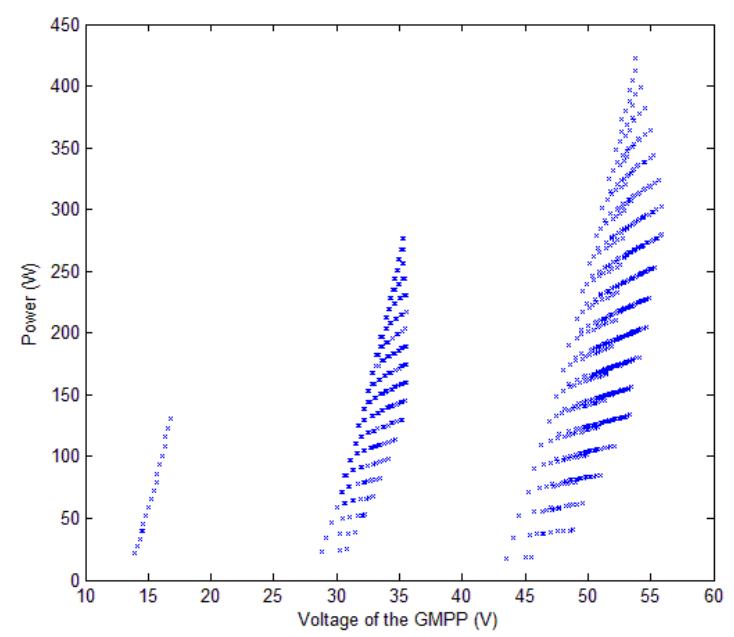

Fig. 3 The Distribution of GMPP

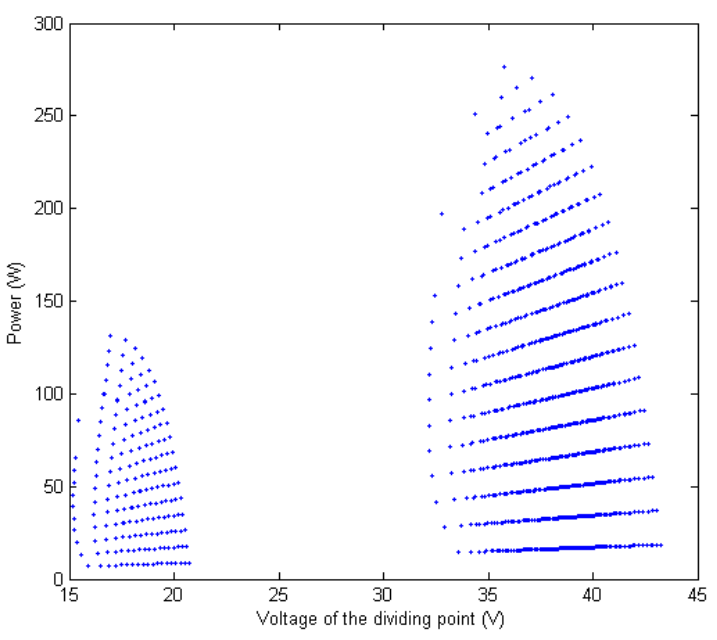

Fig. 4 The Distribution of Dividing Points

\section{The Proposed GMPPT Method}

The Conventional HC Method. Some conventional methods i.e. HC, P\&O and InCond method utilize the feature that the slopes on both sides of the peak point are opposite in sign to track the maximum power point. For example, the HC method perturbs the terminal voltage of the PV system in one direction and observes the change of power. If the change of power is positive, then the method will continue to perturb in the same direction. If the change of power is negative, then the perturbation direction will be reversed. In this way, the operation point can be brought to the peak point. Under PSC when multiple peak points occur in the $\mathrm{P}-\mathrm{V}$ curve, If we set the initial operation point to a point that is 
in a specific part of the P-V curve which is between two dividing points, this method will move the operation point from the initial operation point to the peak point of the same part of the P-V curve. Hence the peak point power of this part of the P-V curve can be obtained. In this way, we can obtain the power of each peak point of the corresponding part of the P-V curve by setting the initial operation point to a point that is on the part. If all the peak point powers are found, the GMPP can be determined. The initial operation point should be in a dividing-point-free region and far from the boundaries of the region.

The Proposed Two-stage GMPPT Method. In view of the behavior of the HC method mentioned above and the distribution of GMPP and dividing points under various conditions, a new two-stage GMPPT method can be proposed. In the first stage, this method manages to find all the possible peak points (if the point exists) and figure out the peak point with the highest power. This is done with the method below. First, set the initial operation point to a point which is in the first dividing-point-free region. Then the $\mathrm{HC}$ method is used to track the peak point in the corresponding part of the P-V curve. This peak point can either be a GMPP or a LMPP. When the peak point is tracked, the voltage and power of the peak point are recorded as Vm and Pm. Next, the method will continue to set the initial operation point to a point which is in the next dividing-point-free region and use the HC method to track the peak point on the next part of the $\mathrm{P}-\mathrm{V}$ curve. The power of the peak point $\mathrm{P}$ is compared to the recorded $\mathrm{Pm}$. If $\mathrm{P}$ is greater than $\mathrm{Pm}, \mathrm{Pm}$ will be changed to $\mathrm{P}$ and $\mathrm{Vm}$ will be changed to the corresponding voltage of $\mathrm{P}$. The procedure mentioned above is repeated until all the possible peak points are compared so that the GMPP is obtained. In the second stage, the method sets the operation point voltage to $\mathrm{Vm}$ and the $\mathrm{HC}$ method is again used to keep the operation point at the GMPP. The operation point power is compared to $\mathrm{Pm}$ at the same time. When shading or irradiance conditions are changed, the operation point power differs much from Pm and this method will back to the first stage so as to restart the peak point searching process. The diagram of the method is shown in Fig. 5.

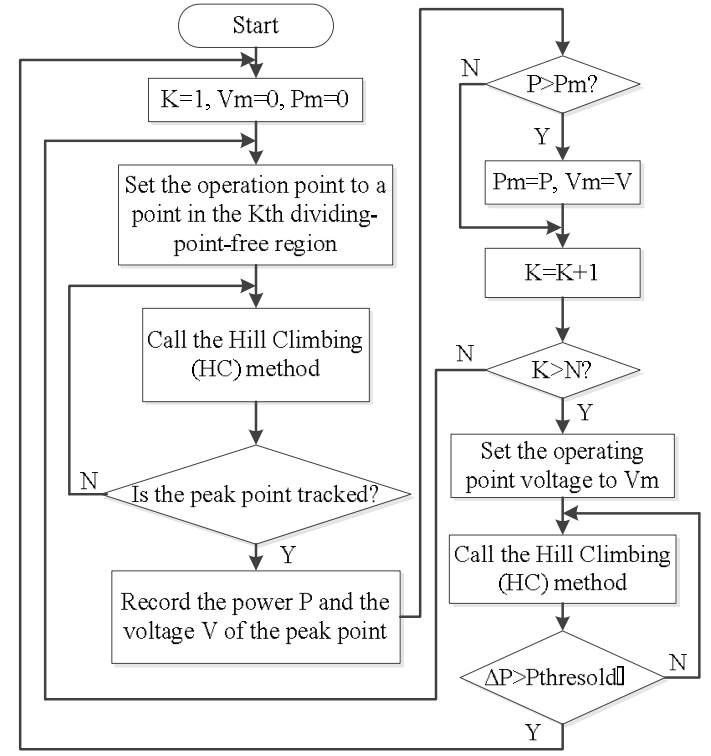

Fig. 5 Diagram of the Proposed Method

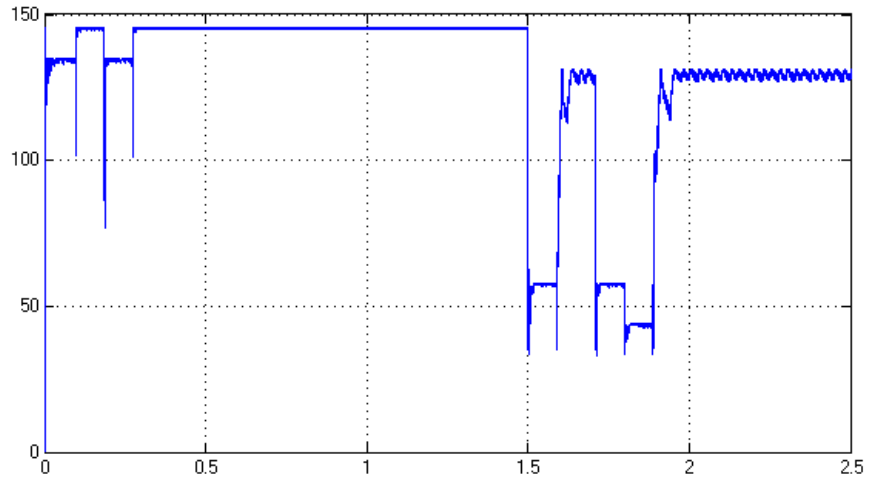

Fig. 6 Simulation Result (P vs. t)

\section{Simulation of the Proposed Method}

To illustrate and verify the workings of this method, we constructed a boost converter connected to the PV system mentioned in the second section. The parameters of the boost converter are shown in Table 2. We used the MPPT controller with our proposed GMPPT method to control the boost converter. At $\mathrm{t}=0 \sim 1.5 \mathrm{~s}$, the irradiances were $[\operatorname{irr} 1, \operatorname{irr} 2, \operatorname{irr} 3]=\left[1000 \mathrm{~W} / \mathrm{m}^{2}, 500 \mathrm{~W} / \mathrm{m}^{2}, 300 \mathrm{~W} / \mathrm{m}^{2}\right]$. The P-V curve of the PV system is shown in Fig. 2(b) and the GMPP was the second peak point. The GMPPT controller succeeded in finding the power of all the peak points which were $131 \mathrm{~W}, 145 \mathrm{~W}$ and $134 \mathrm{~W}$ respectively and found that the second peak point was the GMPP. At $\mathrm{t}=0.28 \mathrm{~s}$, the GMPP was tracked. At $\mathrm{t}=1.5 \mathrm{~s}$, the irradiance condition was changed to imitate a change in shading patterns. The irradiances at 
$\mathrm{t}=1.5 \sim 3.0 \mathrm{~s}$ were $[\operatorname{irr} 1, \operatorname{irr} 2, \operatorname{irr} 3]=\left[1000 \mathrm{~W} / \mathrm{m}^{2}, 200 \mathrm{~W} / \mathrm{m}^{2}, 100 \mathrm{~W} / \mathrm{m}^{2}\right]$. The P-V curve of the PV system is shown in Fig. 2(c) and the GMPP was the first peak point. At $t=1.6 \mathrm{~s}$ the controller noticed a change in power from $145 \mathrm{~W}$ to $57.5 \mathrm{~W}$ then it backed to the first stage to restart the peak point searching process. The controller found that the power of the 3 peak points were $129 \mathrm{~W}, 57.5 \mathrm{~W}$ and $43.5 \mathrm{~W}$ respectively and found that the GMPP is the first peak point. At $\mathrm{t}=1.96 \mathrm{~s}$, the new GMPP was tracked. The power $(\mathrm{P})$ vs. time $(\mathrm{t})$ plot is shown in Fig. 6.

\begin{tabular}{cc} 
Table 2 The Parameters of the Boost & Converter \\
\hline Parameter & Value \\
\hline L1 & $10 \mathrm{mH}$ \\
C1 & $100 \mathrm{uF}$ \\
Output Voltage & $100 \mathrm{~V}$ \\
Switching Frequency & $2 \mathrm{KHz}$ \\
\hline
\end{tabular}

\section{Conclusion}

Based on the distribution of GMPP and dividing points under various conditions, the paper presents a new two-stage GMPPT method. In the first stage, this method moves the operation point to certain regions and uses the HC method to find the peak power point of each region. The GMPP is found after all the possible peak points are found. Then, the method enters the second stage to set the operation point to the GMPP and uses the HC method to keep the operation point at the GMPP. To verify this method, simulation is performed. The simulation result shows that this method can track the true GMPP under PSC in the presence of multiple peak points on the P-V curve of the PV system and can also track the GMPP when conditions are changed. This method is effective in tracking the GMPP and is easy for implementation. This method can be applied to various PV power generation systems.

\section{References}

[1] L. Jia, K. Chen, G. Li, L. Feng, X. Jiang "Output Characteristics and MPPT research for PV array under Partially Shaded Condition", PRZEGLAD ELEKTROTECHNICZNY, ISSN 0033-2097, R. 89 NR 1b/2013.

[2] T. Esram and P. L. Chapman "Comparison of photovoltaic array maximum power point tracking techniques", IEEE Trans. Energy Convers., vol. 22, no. 2, pp.439 -449 2007.

[3] H. Radwan, O. Abdel-rahim, M. Ahmed and M. Orabi "Two Stages Maximum Power Point Tracking Algorithm for PV Systems Operating under Partially Shaded Conditions", International Middle East Power Systems Conference MEPCON2010, pp.683 -688.

[4] N. A. Ahmed and M. Miyatake "A novel maximum power point tracking for photovoltaic applications under partially shaded insolation conditions", Electr. Power Syst. Res., vol. 78, no. 5, pp.777 -784 2008.

[5] K. Kobayashi , I. Takano and Y. Sawada "A study of a two stage maximum power point tracking control of a photovoltaic system under partially shaded insolation conditions", Sol. Energy Mater. Sol. Cells, vol. 90, no. 18/19, pp.2975 -2988 2006.

[6] L. L. Jiang, D. R. Nayanasiri, D. L. Maskell and D. M. Vilathgamuwa "A simple and efficient hybrid maximum power point tracking method for PV systems under partially shaded condition", The 39th Annual Conference of the IEEE Industrial Electronics Society, Vienna, Austria, pp.1513 -1518.

[7] Guo, Guifang, Xiaolan Wu, Shiqiong Zhou, and Binggang Cao. "Modeling of solar photovoltaic cells and output characteristic simulation based on Simulink." Journal of Chemical \& Pharmaceutical Research 6, no. 7 (2014). 